\title{
Hedgehog/Ras interactions regulate early stages of pancreatic cancer
}

\author{
Marina Pasca di Magliano, ${ }^{1}$ Shigeki Sekine, ${ }^{1}$ Alexandre Ermilov, ${ }^{2}$ Jenny Ferris, ${ }^{2}$ \\ Andrzej A. Dlugosz, ${ }^{2}$ and Matthias Hebrok ${ }^{1,3}$ \\ ${ }^{1}$ Diabetes Center, Department of Medicine, University of California, San Francisco, California 94143, USA; ${ }^{2}$ Department \\ of Dermatology and Comprehensive Cancer Center, University of Michigan, Ann Arbor, Michigan 48109, USA
}

\begin{abstract}
Pancreatic ductal adenocarcinoma (PDA) constitutes a lethal disease that affects $>30,000$ people annually in the United States. Deregulation of Hedgehog signaling has been implicated in the pathogenesis of PDA. To gain insights into the role of the pathway during the distinct stages of pancreatic carcinogenesis, we established a mouse model in which Hedgehog signaling is activated specifically in the pancreatic epithelium. Transgenic mice survived to adulthood and developed undifferentiated carcinoma, indicating that epithelium-specific Hedgehog signaling is sufficient to drive pancreatic neoplasia but does not recapitulate human pancreatic carcinogenesis. In contrast, simultaneous activation of Ras and Hedgehog signaling caused extensive formation of pancreatic intraepithelial neoplasias, the earliest stages of human PDA tumorigenesis, and accelerated lethality. These results indicate the cooperation of Hedgehog and Ras signaling during the earliest stages of PDA formation. They also mark Hedgehog pathway components as relevant therapeutic targets for both early and advanced stages of pancreatic ductal neoplasia.
\end{abstract}

[Keywords: Pancreatic cancer; Hedgehog; Kras, mouse model]

Supplemental material is available at http://www.genesdev.org.

Received July 18, 2006; revised version accepted September 25, 2006.

Pancreatic ductal adenocarcinoma (PDA) is the fourth leading cause of cancer death in the United States (Hezel et al. 2006). Annually, its incidence closely matches its mortality, highlighting the inefficacy of existing treatment options (Jemal et al. 2006). Presently, surgical resection is the most effective therapeutic intervention, resulting in survival rates of $\sim 20 \%$ over $5 \mathrm{yr}$; however, most patients are diagnosed at advanced stages when removal of the cancer is not possible.

Pancreatic intraepithelial neoplasias (PanINs) constitute the most common precursor lesions of PDA. PanIN lesions are classified based on histological changes and this histological classification correlates well with the presence of characteristic genetic alterations (Hruban et al. 2001), including mutations in KRAS, INK4A, TP53, and MADH4 (Boschman et al. 1994; Klimstra and Longnecker 1994; Hahn et al. 1996; Rozenblum et al. 1997; Wilentz et al. 2000; Maitra et al. 2003). Early PanINs (PanIN1A/B) are characterized by the change from a cuboidal duct epithelium to cells with columnar shape and by the accumulation of abundant supranuclear mucin. Higher-grade PanINs (PanIN2) show increasing alterations in the cellular architecture, including changes in cell polarity and nuclear atypia. PanIN3 lesions are con-

${ }^{3}$ Corresponding author.

E-MAIL mhebrok@diabetes.ucsf.edu; FAX (415) 564-5813.

Article is online at http://www.genesdev.org/cgi/doi/10.1101/gad.1470806. sidered carcinoma in situ. The cellular changes observed in these tumors are similar to invasive carcinoma; however, cells do not invade through the basement membrane.

Previous studies by us and others (Berman et al. 2003; Thayer et al. 2003) have identified activation of the Hedgehog signaling pathway as a key event in the histogenesis of pancreatic cancer. The expression of the Hedgehog ligands SONIC and INDIAN HEDGEHOG $(\mathrm{SHH}, \mathrm{IHH})$, the transcriptional target gene PATCHED (PTC), and the essential pathway component SMOOTHENED (SMO) is undetectable in normal human pancreatic ducts. In contrast, a relative increase in the expression of these proteins is observed during pancreatic ductal tumorigenesis. Evidence for a causative role of Hedgehog signaling in the development of PanIN lesions comes from studies of transgenic mice in which the Hedgehog ligand Shh is expressed in developing pancreas under the control of promoter elements of the pancreatic and duodenal homeobox gene 1 (Pdx1-Shh) (Thayer et al. 2003). Numerous metaplastic ducts are found in the pancreatic remnant, an indication that pancreas-wide deregulation of the pathway initiates the early steps of tumorigenesis. However, ectopic expression of the soluble Shh protein disrupts the epithelialmesenchymal signaling events essential for proper pancreas organogenesis (Apelqvist et al. 1997; Thayer et al. 2003). Consequently, pancreas formation and function is 
severely affected in $P d x$-Shh mice, resulting in a shortened life span of less than a month after birth. Thus, while important in providing initial evidence for a role of Hedgehog signaling in the development of early precursor lesions, the severe changes in organ morphogenesis and the limited life span of the transgenic mice prohibits long-term studies to test whether continued Hedgehog deregulation is sufficient to cause and maintain pancreatic cancer in adult mice.

Here, we describe the generation of a novel mouse model of pancreatic cancer based on epithelium-specific activation of the Hedgehog signaling pathway. In this model, a dominant active form of the GLI2 transcription factor (CLEG2 transgene), a downstream mediator of the Hedgehog signaling pathway, is conditionally expressed in the pancreas upon Cre-mediated recombination. Tissue-specific activation of the transgene is achieved by crossing CLEG2 transgenic animals with Pdx1-Cre mice (Gu et al. 2002). In contrast to the previously analyzed $P d x$-Shh mice (Thayer et al. 2003), activation of the Hedgehog signaling pathway in $P d x$-Cre; CLEG2 transgenics is cell autonomous and confined to the epithelium. As a consequence, disruption of epithelial-mesenchymal signaling during pancreas development, a hallmark of ectopic Hedgehog ligand expression, is not observed in Pdx1-Cre;CLEG2 mice. Transgenic mice survive into adulthood without impaired exocrine and endocrine pancreas function. However, $30 \%$ of these animals develop pancreatic cancer. These tumors appear undifferentiated and do not form via PanIN lesions commonly found in human patients, suggesting that additional changes are required for the occurrence of these preneoplastic lesions. The vast majority of human pancreatic adenocarcinoma carry signature mutations in the KRAS gene, and expression of a mutated version of Kras, Kras $^{G 12 D}$, recapitulates PanIN progression and PDA formation in mice (Aguirre et al. 2003; Hingorani et al. 2003, 2005). Simultaneous activation of Hedgehog and Ras signaling in triple-transgenic Pdx1-Cre;CLEG2; Kras ${ }^{G 12 D}$ animals results in formation of extensive preneoplastic lesions and significantly reduces the latency of onset of tumor formation and reduces the life span when compared with the double-transgenic Pdx1-Cre;CLEG2 and Pdx1-Cre; Kras ${ }^{G 12 D}$ mice. Thus, although activation of Hedgehog signaling alone does not recapitulate all aspects of human pancreatic adenocarcinoma formation, increased Hedgehog signaling dramatically promotes formation of PanIN lesions in the presence of deregulated Ras signaling. These results shed light on the role of Hedgehog signaling during transformation of pancreatic epithelial cells and the early stages of pancreatic carcinogenesis.

\section{Results}

Epithelial-specific activation of the Hedgehog signaling pathway in the pancreas

We have used a new mouse model of cell-autonomous activation of Hedgehog signaling, the CLEG2 mouse, to conditionally activate the Hedgehog signaling pathway in pancreatic epithelium via ectopic expression of a dominant active version of GLI2, one of the transcriptional mediators of Hh signaling. In the absence of Cre recombinase, the constitutively active CAG promoter induces the expression of a green fluorescent protein (EGFP). The EGFP cDNA is flanked by loxP sites and includes a poly $A$ tail with a strong termination sequence that prevents expression of the downstream myc-GLI2 fusion protein (Fig. 1A). The dominant active version of GLI2, GLI2 $\Delta \mathrm{N}$, consists of an N-terminally truncated version of the protein that lacks an $\mathrm{N}$-terminal repressor domain (Roessler et al. 2005). The GLI2 $\Delta \mathrm{N}$ protein is tagged by a short fragment of the myc protein, a modification that allows detection of the fusion protein via staining with a myc-specific antibody. Upon Cre recombination, the EGFP cassette is excised and the GLI2AN transgene is irreversibly activated in Cre-expressing cells and all of their progeny (Fig. 1B). To determine the consequences of Hedgehog activation in pancreatic epithelium, we have crossed the CLEG2 transgenic animals with $P d x 1$-Cre transgenic mice (Gu et al. 2002). Pancreatic morphology was assessed in 3-wk-old Pdx-Cre; CLEG2 mice. We found that pancreas size, shape, and histology were comparable to control littermates, including wild-type, Pdx-Cre, and CLEG2 single-transgenic mice (Fig. 1C,D; data not shown). This finding is in striking contrast to the loss and disruption of pancreatic tissue previously described in $P d x 1$-Shh mice (Apelqvist et al. 1997).

Recombination of the CLEG2 allele was confirmed by examination of the pancreas under ultraviolet (UV) light. As expected, EGFP expression was readily observed in the pancreas and adjacent organs of CLEG2 single transgenics (Fig. 1E). Efficient Cre recombination of the CLEG2 transgene in Pdx-Cre; CLEG2 mice was indicated by the loss of fluorescence in the pancreas but not in the other organs, including adjacent stomach and duodenal tissues (Fig. 1F; data not shown).

When double-transgenic animals were aged, 30\% developed pancreatic tumors between 6 and 20 wk (Fig. 1G). These animals presented with swollen abdomen, which upon necropsy were determined to be due to tumor mass and hemorrhagic ascites. EGFP-negative tumors were identified both in the head and tail regions of the pancreas (Fig. 1H). As expected, the adjacent organs retained EGFP expression. Although tumors are solitary in most instances, in some cases a second smaller tumor was found within a different area of the pancreas. While all tumors appeared to be locally invasive, no gross metastasis to other organs was observed. Despite the fact that $P d \times 1$ expression has been noted in stomach and duodenum, no recombination of the CLEG2 transgene and no tumors were found in these organs in any of the animals examined. In contrast, some of the Pdx-Cre; CLEG2 animals succumbed to tumors in the cerebellum, most likely due to activity of the $P d \times 1$ promoter element in neural tissue (data not shown). Thus, cell-autonomous activation of Hedgehog signaling within pancreatic epithelium does not disrupt organ development but initiates the formation of pancreatic tumors in adult mice. 
Tumor development in the Pdx-Cre;CLEG2 mice does not progress via PanIN

Older animals presented with pancreatic tumors localized either within the head or the tail of the pancreas (Fig. 2A). Cells within these tumors displayed polygonalto-spindle cell morphology without signs of glandular differentiation. Tumor cells were relatively homogeneous and showed enlarged nuclei and scant cytoplasm (Fig. 2B,C). Areas of necrosis were frequently encountered. Since there were no signs of differentiation toward a specific cell lineage (the tumors did not express insulin, amylase, or $\operatorname{Pdx} 1$ ), we diagnosed these tumors as undifferentiated carcinoma. Ductal proliferation, accompanied by acinar cell loss, was observed in some areas that did not show evident cellular atypia (Fig. 2D).

The expression of the GLI2 $\Delta N$ transgene was confirmed by immunohistochemical staining using an antimyc antibody that recognizes the myc tag of the GLI2myc fusion protein (Fig. 2E). Interestingly, despite the semiquantitative nature of immunostaining, varying expression levels of the GLI $\Delta \Delta \mathrm{N}$ protein were detected. With the exception of a few scattered cells that stained strongly positive for the fusion protein, low-level expres-

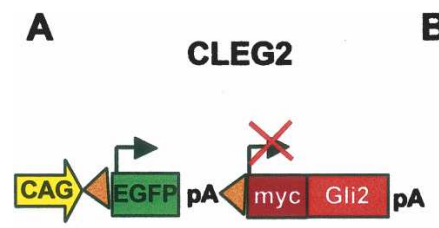

\section{Pdx-Cre;CLEG2}
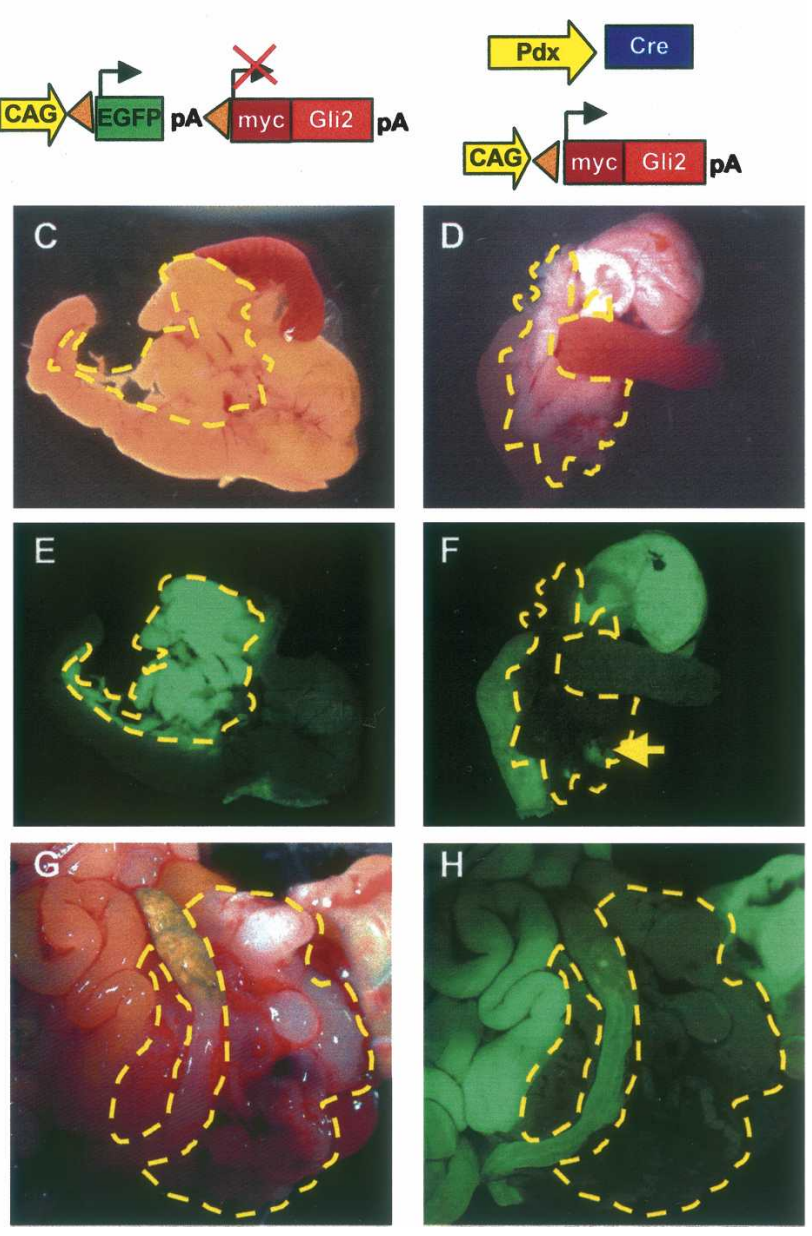

sion was found in the areas of the pancreas that were morphologically normal. In contrast, moderate to high expression levels of the transgene were observed in the tumors and areas of ductal proliferation adjacent to tumors (Fig. 2E). Quantitative PCR analysis revealed that the variable expression levels of GLI2-myc protein are due to differences in GLI2 $\Delta N$ expression. Transcription of transgene mRNA is very low in the nonneoplastic pancreas of Pdx-Cre;CLEG2 mice, while it is expressed at high levels in tumors (data not shown). These differences in mRNA expression indicate either differential activity of the CAG promoter or a distinct mechanism by which transgenic mRNA is stabilized in the tumor cells.

CK19, a cytokeratin expressed in the ductal cell lineage, was undetectable in the undifferentiated tumors (Fig. 2F), a finding that is consistent with what has previously been observed in pancreatic tumors of transgenic mice marked by increased Kras activity (Hingorani et al. 2003). Interestingly, the undifferentiated tumors showed expression of the mesenchymal marker vimentin, while epithelial markers were lost (Hoorens et al. 1998; data not shown). These results are reminiscent of previous studies in which a transition from epithelial cells toward cells displaying mesenchymal characteristics had been described in tumors derived from pancreatic ductal cells (Deramaudt et al. 2006).

Pancreatic adenocarcinomas are believed to develop

Figure 1. Pdx-Cre;CLEG2 mice develop pancreatic cancer. $(A)$ Schematic of the CLEG2 transgene. In the absence of Cre expression, the constitutively active $C A G$ promoter drives expression of EGFP in multiple organs. Under these conditions a polyA sequence with strong termination signal inhibits transcription of the GLI2 $\Delta N$ transgene located $3^{\prime}$ of the EGFP cDNA. The EGFP cDNA is flanked by loxP sites (triangles). (B) The CLEG2 transgenic mouse was crossed with transgenic $P d \times 1-C r e$ animals to initiate recombination within the whole pancreatic epithelium of $P d x$-Cre;CLEG2 bitransgenic mice. Cre-mediated excision of the floxed EGFP-stop cassette results in transcription of the GLI2 $\Delta N$ transgene. The myc tag present at the $5^{\prime}$ end of the myc/Gli2 $\Delta \mathrm{N}$ fusion protein allows immunohistochemical detection of the transgene. $(C)$ Gross morphology of the pancreas in a control single-transgenic CLEG2 mouse. The pancreas, nestled between the stomach, the spleen, and the duodenum, is outlined with a yellow dashed line. $(D)$ The size and gross morphology of Pdx-Cre;CLEG2 pancreas (outlined in yellow) is comparable to control. $(E)$ Stomach, duodenum, and pancreas express GFP in a CLEG2 mouse. The fluorescence in the pancreas appears especially intense due to the light color and density of the organ. $(F)$ The expression of GFP is retained in the stomach and duodenum of Pdx-Cre;CLEG2 mice, but it is absent from the pancreas, indicating tissue-specific Cre recombination. A fluorescent lymph node within the pancreas is indicated with an arrow. Note that different exposure times were used for $E$ and $F$ due to the strong fluorescence of the pancreas in CLEG2 mice. (G) Tumor (outlined in yellow) invading the abdominal cavity of a 4-mo-old Pdx-Cre;CLEG2 mouse. $(H)$ UV light picture of the same tumor demonstrates absence of EGFP expression, consistent with recombination of the CLEG2 transgene. The surrounding organs remain EGFP positive. 
Figure 2. Tumors in $P d x-C r e$; CLEG2 mice are undifferentiated. (A) A massive tumor (in a 12 wk-old mouse), firmly attached to the gastrointestinal organs, has invaded the abdominal cavity and impaired the digestive process. (B) Histological section through a representative tumor (the tumor border is marked by a dashed yellow line). Note the prominent vascular space $(\mathrm{v})$ and the pronounced necrotic area $(\mathrm{n}) .(C)$ High magnification of tumor cells. Tumor cells are undifferentiated and have enlarged nuclei and scant cytoplasm. Ductal structures are not observed. $(D)$ Acinar-ductal replacement is observed in the periphery of the tumor (white arrows). The tumor border is marked by a yellow dashed line. Note the presence of remnants of acinar cells within the ducts. (E) Immunohistochemistry for myc. Tumor cells show strong nuclear staining (outlined in yellow). A subset of cells in ducts undergoing acinar-ductal replacement exhibits moderate level of myc expression (arrows). (F) Staining for CK19. Tumor tissue (border marked in yellow) is negative, whereas a duct adjacent to the tumor is strongly positive.
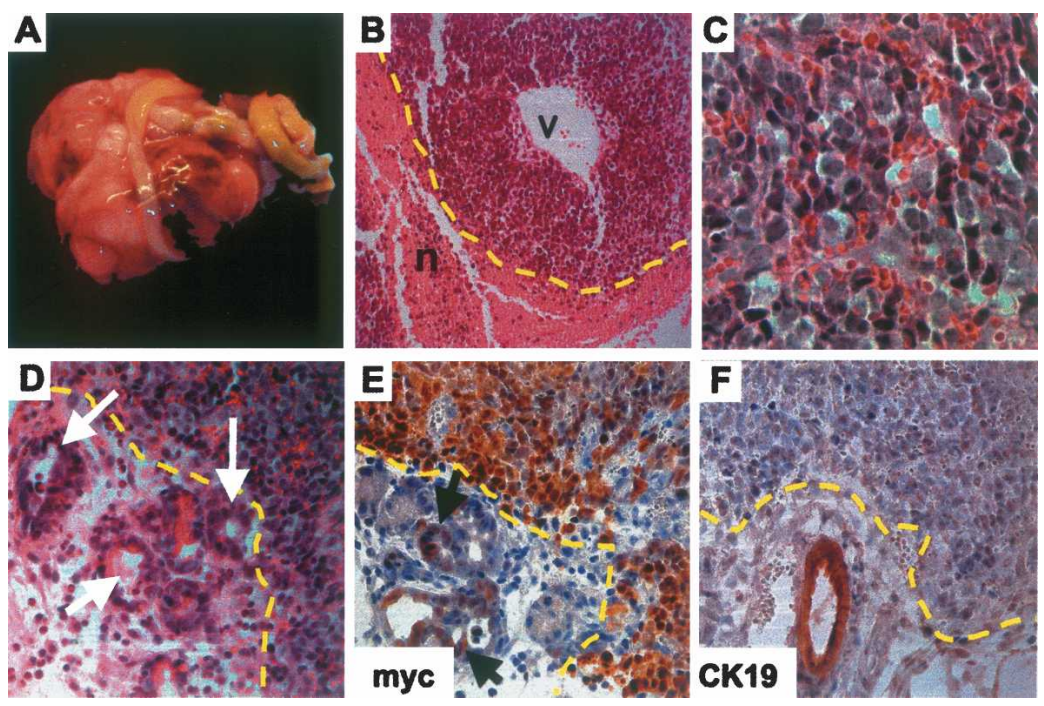

from pancreatic ducts, and initial lesions commonly progress through well-characterized PanINs (Hruban et al. 2001). However, we could not detect any noninvasive neoplastic lesions in these mice as defined by the Penn Workshop (Hruban et al. 2006). Thus, tumor formation upon epithelial-specific Hedgehog activation does not progress via classical PanIN lesions. These findings suggest that other signaling changes are required to more completely recapitulate the human disease.

\section{Activation of the CLEG2 transgene in the context of Kras mutation results in extensive PanIN formation and increased mortality}

The vast majority of human pancreatic tumors are marked by mutations in the KRAS gene that render the protein independent of regulatory mechanisms (Hezel et al. 2006). The significance of increased Kras signaling in pancreatic cancer is supported by the observation that conditional expression of $\operatorname{Kras}^{G 12 D}$, the most common mutation in human tumors, results in formation of PanIN lesions and pancreatic tumors in transgenic $P d x$ Cre; Kras $^{G 12 D}$ mice (Aguirre et al. 2003; Hingorani et al. 2003, 2005). However, in the absence of additional mutations in other tumor suppressors or oncogenes the lesions progress slowly and only a minority of these mice develop invasive tumors (Hingorani et al. 2003).

In humans, deregulation of the Hedgehog signaling pathway has been noted in PanIN1 lesions (Thayer et al. 2003), suggesting that ectopic Hedgehog activation might provide the "second hit" that precipitates tumor formation in Pdx-Cre; Kras ${ }^{G 12 D}$ mice. To test this hypothesis, we crossed the Pdx-Cre;CLEG2 and Kras ${ }^{G 12 D}$ mice to generate triple-transgenic Pdx-Cre;CLEG2;Kras ${ }^{G 12 D}$ animals. While the pancreas appeared normal at birth (Supplementary Fig. 1), the health of all triple-transgenic mice $(n=18)$ deteriorated rapidly and the animals were sacrificed between 3 and 8 wk of age (Supplementary Fig. 2). In all animals, the pancreas appeared fibrotic at necropsy, with some animals presenting with grossly detectable tumors and hemorrhagic ascites. Pancreata of $P d x$ Cre; CLEG2; Kras ${ }^{G 12 D}$ mice invariably showed characteristic histological features. Normal organization of pancreas architecture was completely disrupted and replaced by proliferation of ducts exhibiting lobular structures against a background of extensive fibrosis (Fig. 3C). Acinar cells were almost completely abolished. These dramatic changes in tissue architecture of $P d x$ Cre;CLEG2; Kras ${ }^{G 12 D}$ mice were in stark contrast to the apparently normal pancreas morphology found in $P d x$ Cre;CLEG2 (Fig. 3A) and Pdx-Cre;Kras ${ }^{G 12 D}$ (Fig. 3B) mice at this stage. The ductal epithelium showed varying degrees of atypia, corresponding to PanIN1A-3 (Fig. 3D-F). In some areas, epithelial cells showed abundant cytoplasmic mucin and basally located small nuclei (Fig. 3D). In other areas, ductal cells exhibited enlarged atypical nuclei and pronounced papillary projections (Fig. 3F). Most of the cells within the enlarged ducts found in PanIN lesions express CK19 (Fig. 3G); however, some cells seemed to have lost their polarity within the duct epithelium and ceased to express CK19 (Fig. 3G, arrowheads). As observed in human patients, PanINs expressed abundant mucins as shown by alcian blue and PAS staining (Fig. 3H,I). The expression of the GLI2/myc fusion protein in the enlarged ducts was confirmed via immunohistochemistry with an anti-myc antibody (Fig. 3J). Similarly to Pdx-Cre;CLEG2 mice, PdxCre;CLEG2;Kras ${ }^{\text {G12D }}$ mice developed undifferentiated carcinomas composed of tumor cells with polygonal-to-spindle morphology and areas of necrosis (Fig. 3K,L). In a few mice, stromal invasion of differentiated tumors was suspected. Thus, while the PanINs observed in PdxCre;CLEG2;Kras ${ }^{\mathrm{G} 12 \mathrm{D}}$ 

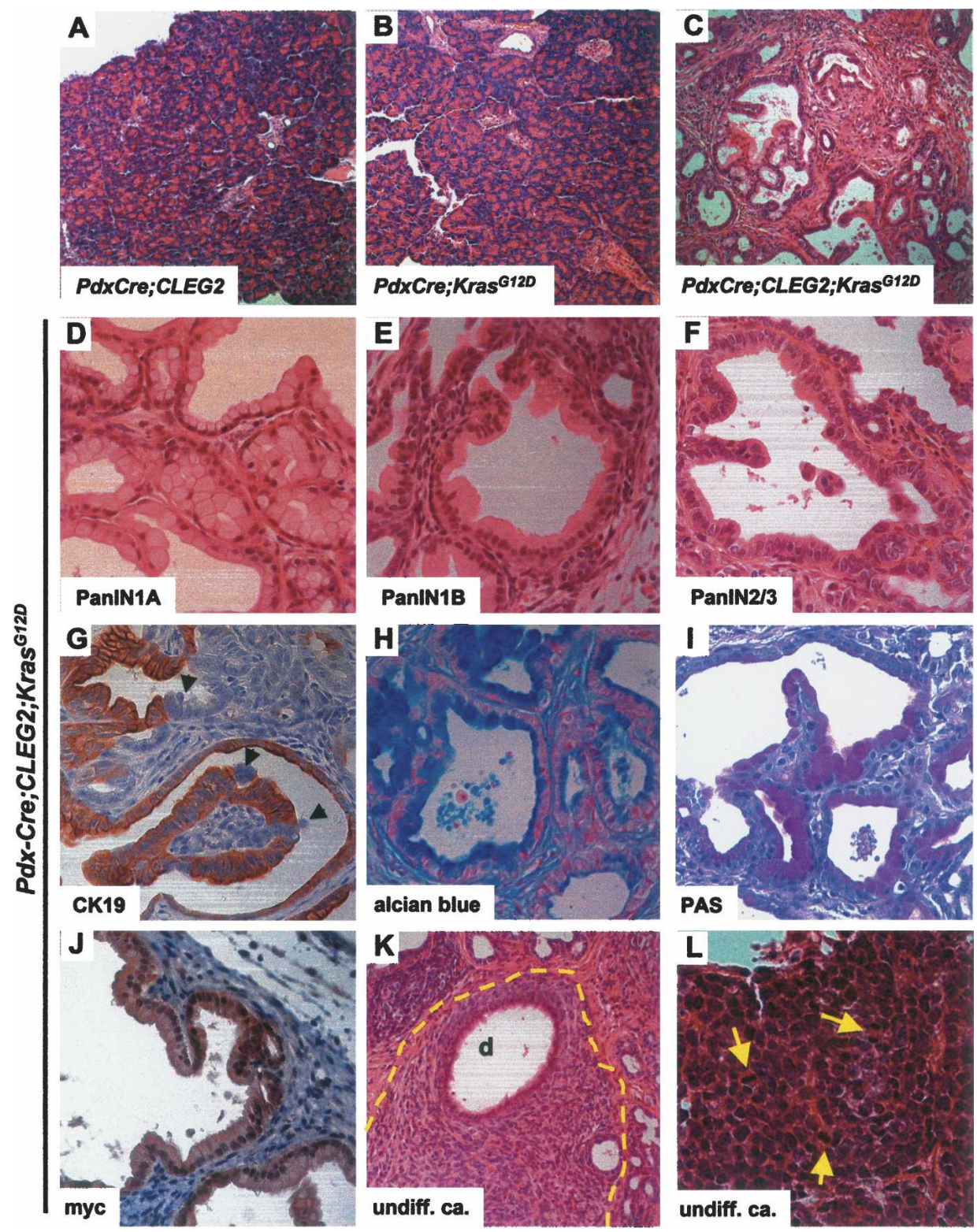

Figure 3. Histology of lesions and tumors observed in Pdx-Cre;CLEG2; Kras ${ }^{G 12 D}$ mice. $(A, B)$ Low-magnification images of the pancreas of 6-wk-old Pdx-Cre;CLEG2 $(A)$ and Pdx-Cre;Kras ${ }^{G 12 D}(B)$ mice. The composition and structure of the pancreas appears normal. $(C)$ Low-magnification image showing the pancreas histology of a 6-wk-old Pdx-Cre;CLEG2;Kras ${ }^{G 12 D}$ mouse. The normal pancreas morphology has been replaced by an extensive network of dilated ducts that are interspersed by fibrotic areas. Acinar cells that constitute the majority of cells in a normal pancreas are almost completely lost. $(D-F)$ Formation of PanIN lesions in $P d x$ Cre;CLEG2; Kras ${ }^{G 12 D}$ pancreata. (D) PanIN1A lesions composed of characteristic goblet cells with basal nuclei and abundant intracellular mucin. (E) PanIN1B lesions. The epithelial cells show somewhat enlarged nuclei. (F) PanIN2/PanIN3 lesions with papillary growth and higher nuclear/cytoplasmic ratio of the tumor cells. $(G)$ Most duct cells within PanIN lesions are positive for CK19. Subsets of cell clusters have lost CK19 expression and show altered polarity (arrowheads). $(H, I)$ Epithelial cells within PanIN lesions show mucin accumulation as marked by alcian blue $(H)$ and PAS $(I)$ stainings. (J) Detection of the GLI2 $\Delta \mathrm{N} / \mathrm{myc}$ fusion protein in a PanIN lesion by immunohistochemistry with an anti-myc-tag antibody. $(K, L)$ Invasive tumors. $(K)$ Undifferentiated carcinoma (outlined in yellow). Polygonal-to-spindle tumor cells show solid growth and surround a duct (d). (L) High-magnification picture of tumor cells. Tumor cells with high nuclear/cytoplasmic ratio form a solid pattern. Mitotic figures are indicated by arrows.

mice strongly resemble those found in human patients, the predominantly undifferentiated tumors found in the transgenic pancreata are distinct from human PDA (a schematic depicting the various tumor types is shown in Supplementary Fig. 3).
The endocrine compartment appeared normal in tumor-bearing Pdx-Cre;CLEG2;Kras ${ }^{G 12 D}$ transgenic animals by histological inspection (data not shown), possibly reflecting the importance of cellular context in determining responsiveness to oncogenic stimuli. Alterna- 
tively, the CAG promoter might be less active in islet cells, resulting in lower Hedgehog activity in these cells. The notion of functional pancreatic endocrine cells is supported by the fact that transgenic mice maintain normal blood glucose levels for the first few weeks of life (blood glucose levels decreased significantly in the final stages of the disease).

Tumors in Pdx-Cre;CLEG2 and Pdx-Cre;CLEG2;Kras ${ }^{\mathrm{G} 12 \mathrm{D}}$ animals share common marker expression

A number of experiments were performed to define the molecular mechanisms that mediate the formation of pancreatic carcinoma in Pdx-Cre;CLEG2 and $P d x$ Cre;CLEG2;Kras ${ }^{G 12 D}$ tumor models. As expected, precursor lesions and tumors found in both transgenic models have an elevated proliferation index, as determined via ki67 staining (Fig. 4A-E). While few proliferating cells are observed within ducts of nontransgenic con- trols, numerous ki67-positive cells were observed in the abnormal ducts adjacent to tumors. Interestingly, no increase in proliferation was detected in the nontumorigenic exocrine and endocrine compartments /data not shown). Thus, increased proliferation is confined to transformed areas within the pancreas.

Previous studies have shown that up-regulation of the Notch signaling pathway occurs in human pancreatic cancer (Miyamoto et al. 2003). Activity of Notch signaling can be monitored via staining for Hes1, a direct transcriptional target of the pathway. Under normal conditions, Hes1 is expressed early during pancreas formation but is down-regulated at the end of development (Jensen et al. 2000; Lammert et al. 2000). In the adult animals, only the centroacinar and a few duct cells are positive for Hes1 staining (Fig. 4F, arrowheads; Miyamoto et al. 2003; Stanger et al. 2005). A similar pattern of expression was observed in pancreata of young Pdx-Cre;CLEG2 mice before the onset of tumor formation (data not

Figure 4. Characterization of gene expression in lesions and tumors from $P d x-C r e ; C L E G 2$ and $P d x-$ Cre;CLEG2;Kras ${ }^{G 12 D}$ mice. $(A-E)$ Proliferating cells are detected with immunostaining against ki67. (A) Control pancreas. Increased proliferation is observed in both acinar-ductal replacement $(B)$ and tumors $(C)$ in Pdx-Cre; CLEG2 mice. $(D, E) \mathrm{Pa}$ nIN lesion $(D)$ and undifferentiated tumor $(E)$ in Pdx-Cre; CLEG2;Kras ${ }^{G 12 D}$ pancreata. (F) Hes1 labels centroacinar cells but not duct cells in adult pancreas (arrowheads). (d) Duct; (v) blood vessel. $(G, H)$ Elevated Hes1 levels in proliferating ducts $(G)$ and tumor cells $(H)$ in a Pdx-Cre;CLEG2 mouse. $(I)$ Hes1 expression is up-regulated in a PanIN lesions $(I)$ and poorly differentiated tumors $(J)$ in a $P d x$ Cre;CLEG2; Kras ${ }^{G 12 D}$ mouse. (K) Membrane-associated E-cadherin expression in control pancreas. $(L, N)$ Membrane E-cadherin is retained in the areas of acinar-ductal replacement of the Pdx-Cre; CLEG2 mice as well as in PanINs of $P d x$ Cre; CLEG2; Kras ${ }^{G 12 D}$ animals. $(M, O)$ E-cadherin expression is lost in the undifferentiated areas of both Pdx-Cre;CLEG2 and Pdx-Cre;CLEG2;Kras ${ }^{G 12 D}$ tumors.
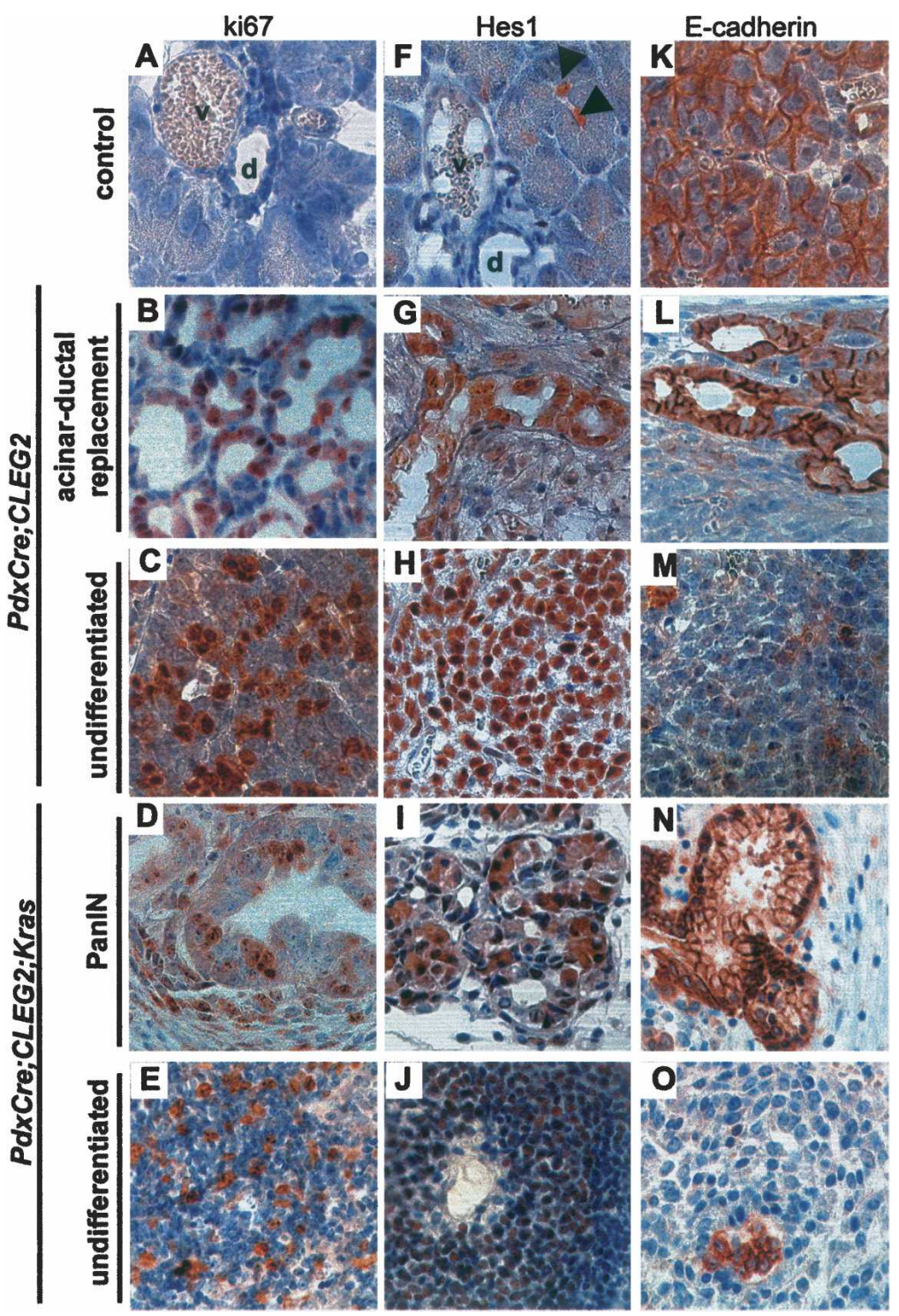
shown). Consistent with the findings in human adenocarcinoma, pancreatic lesions in both the Pdx-Cre; CLEG2 and Pdx-Cre;CLEG2;Kras ${ }^{G 12 D}$ animals displayed up-regulation of Hes1 staining (Fig. 4G-J). It should be noted that, in contrast to PanIN lesions, ducts that appeared normal by histological examination remained Hes1 negative (data not shown).

A characteristic of tumor cells is the loss of normal cell-cell contacts that maintain epithelial integrity. Expression of E-cadherin, a transmembrane protein that ensures cell adhesion properties in epithelial cells, is also displaced from its normal cellular location in the undifferentiated regions of the lesions and tumors in both transgenic models (Fig. 4K-O). Membrane localization of E-cadherin is retained in the areas of acinar-ductal replacement in Pdx-Cre;CLEG2 and in PanIN lesions in Pdx-Cre;CLEG2;Kras ${ }^{G 12 D}$ mice (Fig. 4L,N; data not shown). Thus, activation of Hedgehog signaling either alone or in the context of increased Kras signaling activates proliferation and modulates the differentiation status of epithelial cells.

\section{Ras/Hedgehog interactions}

Previous results from $P d x-S h$ mice had suggested that ectopic expression of Hedgehog ligands is sufficient to activate the Ras signaling pathway by inducing a mutation in the Kras gene (Thayer et al. 2003). However, sequencing of the Kras gene in DNA extracted from $P d x$ Cre;CLEG2 tumor tissue revealed no activating mutations (data not shown). Consistent with this result, we failed to observe a significant increase in phosphorylated ERK1/2, one of the downstream effectors of Ras signaling, in Pdx-Cre;CLEG2 tumors (Fig. 5A). As expected, the activating mutation in Kras present in $P d x$ Cre;CLEG2;Kras ${ }^{G 12 D}$ mice lead to strong phosphoERK1/2 levels in PanIN lesions and tumors (Fig. 5A; data not shown).

Ras signaling activates the phosphoinositide-3-kinase pathway (PI3K). This results in phosphorylation of downstream components, including PDK, AKT, and s6K (p70), all of which are found at elevated levels in both Pdx-Cre;CLEG2 tumors and Pdx-Cre;CLEG2;Kras ${ }^{G 12 D}$ PanIN lesions/tumors (Fig. 5B,C; data not shown). While these results might argue for activation of a specific branch of Ras signaling, previous studies have shown that PI3K can also be activated in a Ras-independent manner downstream from receptor tyrosine kinases. Phosphorylation of one of these tyrosine kinases, the epidermal growth factor receptor (EGFR), is observed in early PanIN lesions and sustained in pancreatic cancer. In support of EGFR-mediated activation of PI3K signaling we found that phospho-EGFR is consistently upregulated in both Pdx-Cre;CLEG2 tumors and Cre;CLEG2;Kras ${ }^{G 12 D}$ PanIN lesions/tumors (Fig. 5C; data not shown).

In summary, these data suggest that cell-autonomous activation of the Hedgehog pathway is not sufficient to induce mutations in the Kras gene or to activate the mitogen-activated protein kinase (MAPK) branch of the Ras signaling cascade. However, other molecular changes implicated in pancreatic cancer formation, including phosphorylation of EGFR and activation of AKT signaling, play roles in the histogenesis of $P d x$-Cre; CLEG2 tumors.

Ras/Hedgehog interactions mediate stromal expansion in Pdx1-Cre;CLEG2;Kras ${ }^{\mathrm{G} 12 \mathrm{D}}$ tumors

Previous studies have noted that Ras signaling can induce Hedgehog signaling during the formation of pancreatic tumors (Hingorani et al. 2005). To determine the level of Hedgehog activity in the different transgenic animals we performed quantitative PCR for Ptc1, a Hedgehog receptor and downstream target gene of Gli. As expected, high Ptc1 expression levels were observed in Pdx-Cre;CLEG2 tumors when compared with control pancreatic tissue (Fig. 5D). Enhanced levels of Ptc1 indicative of active Hedgehog signaling were also detected in Pdx-Cre;CLEG2; Kras ${ }^{G 12 D}$ fibrotic pancreata containing extensive PanIN lesions. Expression levels of Gli1, another target gene of the Hedgehog pathway, were similarly increased in Pdx-Cre;CLEG2 tumors and $P d x$ Cre;CLEG2; Kras ${ }^{G 12 D}$ PanINs (Fig. 5D). These data confirm that activation of the Hedgehog target gene has occurred as a consequence of CLEG2 transgene expression.

Increased expression of Sonic Hedgehog has been noted in human PanIN lesions and in transgenic PdxCre;Kras ${ }^{G 12 D}$ mice that carry an additional mutation in the Trp53 tumor suppressor gene (Hingorani et al. 2005). Interestingly, the transcript levels of the Hedgehog ligands Shh and Ihh are significantly increased in the PanIN lesions of Pdx-Cre;CLEG2;Kras ${ }^{G 12 D}$ but not in $P d x$-Cre;CLEG2 tumor or control tissues (Fig. 5D). Immunohistochemical analysis confirmed expression of Shh in Pdx-Cre;CLEG2;Kras ${ }^{G 12 D}$ PanIN lesions but not in areas of acinar-ductal replacement in $P d x$-Cre;CLEG2 mice (Fig. 5E,H). It should be noted that, in contrast to the PanIN lesions, the undifferentiated tumors in $P d x$ Cre;CLEG2; Kras ${ }^{G 12 D}$ did not show elevated expression of Hedgehog ligands, possibly due to the fact that cancer cells have lost their differentiation status (data not shown). Thus, these results indicate that Ras activation regulates Hedgehog signaling during tumor formation via activation of ligand expression.

Expression of Hedgehog ligands during pancreas development has been shown to stimulate expansion of pancreatic mesenchyme during organogenesis (Kawahira et al. 2005). Gomori trichrome staining of pancreata from Pdx-Cre;CLEG2;Kras ${ }^{G 12 D}$ mice revealed prominent desmoplasia (Fig. 5I), a common feature of pancreatic adenocarcinoma (Hezel et al. 2006). Ki67 staining showed that the desmoplastic stromal cells in PanIN lesions of $P d x$ Cre;CLEG2; ras $^{G 12 D}$ were actively proliferating (Fig. 5J). In contrast, very little stroma with minimal proliferative activity was observed in the tumors from Pdx-Cre; CLEG2 animals that also lack expression of Hedgehog ligands (Fig. 5F,G). Thus, our results correlate the pres- 
Figure 5. Hedgehog/Ras signaling and desmoplasia in Pdx-Cre; CLEG2 and PdxCre;CLEG2; Kras ${ }^{G 12 D}$ mice. (A) Western blot shows activation of the MAPK pathway, measured as elevated levels of phospho-ERK1/2, in the PanIN lesions of $P d x$ Cre;CLEG2; Kras ${ }^{G 12 D}$ mice (K). PhosphoERK1/2 is barely detectable in control pancreas $(\mathrm{p})$ and tumor tissue isolated from Pdx-Cre;CLEG2 mice (C). In all cases, no significant difference is observed in the total amount of ERK1/2. GAPDH (abbreviated as GAP) served as loading control. (B) Western blot analysis shows overexpression of phosphorylated PDK1 and AKT in transgenic tissue compared with control pancreas. The total AKT protein levels are unchanged. $(C)$ Elevated levels of phospho-EGFR (active form) in Pdx-Cre; CLEG2 (C), and Pdx-Cre; CLEG2; Kras ${ }^{G 12 D}(\mathrm{~K})$ animals compared with wild-type pancreas (p). Similarly, phospho-s6 $\mathrm{k}$ is increased in transgenic samples compared with control pancreas. Total AKT and GAPDH protein levels are unchanged. $(D)$ Expression of Hedgehog signaling components. Quantitative PCR shows significantly increased levels of Ptc1 and Gli1 in Pdx-Cre;CLEG2 (C) and in Pdx-Cre;CLEG2;Kras ${ }^{G 12 D}$ mice (K) compared with wild-type pancreas tissue (p). Quantitative PCR analysis of Shh expression confirms that Shh levels are undetectable in normal pancreas and in tumors from Pdx-Cre;CLEG2 mice. In contrast, expression is significantly increased in the PanIN lesions of Pdx-Cre; CLEG2; Kras ${ }^{G 12 D}$ mice. Similarly, Ihh expression is elevated in Pdx-Cre; CLEG2; Kras ${ }^{G 12 D}$ samples (K) but not in Pdx-Cre;CLEG2 (C). Control pancreas samples are labeled as "p." $(E, H)$ Immunohistochemical analysis confirms Shh expression in $P d x$ Cre; CLEG2; $\operatorname{Kras}^{G 12 D}(E)$ but not in Pdx-Cre; CLEG2 acinar-ductal replacement areas or tumors $(H)$. $(F, I)$ Gomori trichrome staining indicates little stromal infiltration in $P d x$-Cre; $C L E G 2$ tumors. Ducts are outlined in yellow. $(F)$ In contrast, extensive fibrosis reaction, marked by green Gomori staining, is observed in mesenchyme surrounding PanIN lesions in Pdx-Cre;CLEG2;Kras ${ }^{G 12 D}$ animals $(I)$. $(G)$ ki67 staining indicates lack of proliferation in the mesenchymal area (outlined in yellow) surrounded by a Pdx-Cre;CLEG2 tumor. Note that ki67-positive cells are found within the tumor tissue (bottom part of the picture). ( $J$ ) In contrast, proliferative activity is observed in the stroma of a Pdx-Cre;CLEG2; Kras ${ }^{G 12 D}$ pancreas. The border of the epithelium is outlined in yellow; some ki67-positive nuclei in stromal cells are indicated by arrows.

ence of Hedgehog ligands with the degree of desmoplasia, and suggest that ligand expression might be required for this change in tumor stroma. These data also suggest that while increased Hedgehog signaling within pancreatic epithelial cells results in pancreatic tumors, development of PanIN lesions indicative of early stages of human pancreatic adenocarcinoma formation may require cooperation between the Ras and Hedgehog pathways.

\section{Discussion}

By modeling the genetic mutations found in human PDA, recent studies in transgenic mice have started to unravel the molecular mechanisms that underlie the initial stages of pancreatic tumor formation. Deregulation of the Hedgehog pathway has been implicated in the initiation and maintenance of numerous tumor types (Pasca di Magliano and Hebrok 2003). Additionally, Hedgehog signaling becomes activated in the earliest PanIN lesions, and the expression level of pathway components increases during the progression toward PDA (Berman et al. 2003; Thayer et al. 2003). Therefore, we decided to test whether sustained Hedgehog activation is sufficient to cause PDA in mice.

To circumvent the disruption of pancreas organogenesis caused by altered mesenchymal-epithelial signaling due to ectopic expression of Hedgehog ligands (Thayer et al. 2003; Kawahira et al. 2005), we decided to cell-autonomously activate the Hedgehog pathway within pancreatic epithelium via Cre-mediated expression of an $\mathrm{N}$ terminally truncated, dominant active version of the 
transcription factor GLI2 (GLI2 $\Delta \mathrm{N}$ ) (Roessler et al. 2005). Hedgehog signaling is mediated via the Gli family of transcription factors that include Gli1-3 (Ruiz i Altaba et al. 2002). Gli2 was chosen because it is believed to be the main transactivator of the pathway, in contrast to Gli1, which appears to play a minor role in pathway activation, and Gli3, which usually functions to inhibit Hedgehog signaling. Gli1, however, is a transcriptional target of the pathway, and measuring Glil mRNA expression level can be used as a readout of Hedgehog activity. As expected, GLI2 overexpression results in activation of Glil transcription in the transgenic Pdx-Cre; CLEG2 mice. Pancreas development in the Pdx-Cre; CLEG2 mice is normal by gross morphological inspection and transgenic animals survive to adulthood; however, a significant percentage of these mice develop pancreatic tumors. Surprisingly, tumor formation does not appear to follow the classical PDA progression model through PanIN. Furthermore, the invasive tumors are different from PDA as they present with undifferentiated histology. Thus, our results suggest that ectopic activation of Hedgehog signaling in the pancreatic epithelium is sufficient to cause cellular transformation but does not promote PDA formation via a step-wise progression involving PanIN lesions at early stages.

\section{Cooperation between Hedgehog and Ras signaling during PanIN formation}

Hedgehog signaling can provide an oncogenic stimulus in a number of organs, and increased pathway activation during PanIN formation in the progression toward human PDA indicates that it may play an important role in pancreatic tumorigenesis. The lack of PanIN and PDA formation in $P d x$-Cre; CLEG2 mice suggests the absence of additional signals essential for PanIN progression. Our results show that deregulation of the Ras pathway can provide these signals. Increased Ras signaling by itself results in PanIN lesions with a long latency. Up to 4.5 mo of age, most ducts in Pdx-Cre; Kras ${ }^{G 12 D}$ transgenic mice are normal or present with minimal PanIN1A lesions, and more advanced lesions only become prevalent when mice reach 9 mo of age (Hingorani et al. 2003). Strikingly, simultaneous activation of both the Hedgehog and Ras pathways significantly reduces the latency of PanIN formation. By the time triple-transgenic mice reach 3 wk of age, the normal pancreas architecture is almost completely substituted by PanIN lesions that are surrounded by abundant mesenchyme. Furthermore, the triple-transgenic Pdx-Cre;CLEG2;Kras ${ }^{G 12 D}$ mice have a significantly shortened life span due to increased tumor burden when compared with the Pdx-Cre;CLEG2 and Pdx-Cre;Kras ${ }^{G 12 D}$ double-transgenic mice. The fact that most tumors are undifferentiated might indicate that cell-autonomous activation of Hedgehog signaling severely disrupts the differentiation status of the targeted cells.

How does Ras signaling control PanIN formation in Pdx-Cre;CLEG2;Kras ${ }^{G 12 D}$ mice? During PanIN progression in humans, the increase in Hedgehog signaling ac- tivity-as marked by the elevated expression of the Hedgehog receptor and transcriptional target, PTC1-is concomitant with the increased expression of Hedgehog ligands, including SHH (Thayer et al. 2003). Interestingly, deregulation of Ras signaling in mice lacking p53 function in the pancreatic epithelium has recently been shown to induce Shh expression (Hingorani et al. 2005). These results suggest that Kras activation and/or elimination of p53 function is sufficient to cause expression of Shh in pancreatic tissue. Given the fact that KRAS mutations appear significantly earlier than TP53 mutations during human PanIN progression, it is likely that deregulation of Ras activity is responsible for the induction of $\mathrm{SHH}$ expression in human pancreatic cancer. Here we show that Pdx-Cre;CLEG2;Kras ${ }^{G 12 D}$ but not PdxCre;CLEG2 mice initiate Hedgehog ligand expression. These findings are important as they provide evidence for the notion that deregulation of Ras signaling during the earliest PanIN stages in humans is sufficient to induce Hedgehog signaling. Moreover, this induction occurs via expression of Hedgehog ligands and thus does not require pathway activation caused by mutations in Hedgehog signaling components. In support of this hypothesis, mutations in genes that deregulate Hedgehog activity and consequently cause tumors in other tissues have not been identified in pancreatic cancer specimens.

Unfortunately, forced expression of Hedgehog ligands severely disrupts pancreas development and results in early postnatal lethality (Apelqvist et al. 1997). In the absence of transgenic mice that would allow temporally and spatially appropriate expression of Hedgehog ligands in the mature pancreas, we can only speculate on the exact role that elevation of these factors might play during PanIN formation. Pancreatic adenocarcinoma is characterized by desmoplasia, the presence of a dense population of fibroblasts and inflammatory cells (Hezel et al. 2006). It is noteworthy that desmoplasia is present in Pdx-Cre;CLEG2; Kras ${ }^{G 12 D}$ PanINs but lacking in $P d x$ Cre;CLEG2 mice. Interestingly, ectopic expression of Shh has been shown to increase the mesenchymal compartment within the developing pancreas (Kawahira et al. 2005). Thus, Hedgehog ligand expression could be responsible for stromal infiltration during tumor formation. However, our current results do not establish a causative relation between increased Hedgehog ligand expression and desmoplasia, as other signals could also mediate stromal infiltration.

\section{Absence of Kras mutations in Pdx-Cre;CLEG2 mice}

The comparison of distinct models of pancreatic cancer provides the opportunity to define genetic lesions involved in the earliest stages of PDA initiation. Signature activating mutations in codon 12 of KRAS are almost invariable in human PDA tumor specimens and occur as early as PanIN1A lesions (Almoguera et al. 1988; Moskaluk et al. 1997). Our work has implicated deregulation of Hedgehog signaling as another pathway sufficient to cause transformation of pancreatic epithelial 
cells. These results raise the question of whether deregulation of the Hedgehog pathway could activate Ras signaling to initiate cellular transformation. Analysis of pancreatic lesions in $P d x$-Shh mice suggested that ligand-induced Hedgehog signaling is sufficient to cause activating mutations within the Kras gene (Thayer et al. 2003). In contrast to these data, we failed here to detect activating mutations in Kras or a general elevation of Ras signaling upon epithelial-specific activation of Hedgehog signaling in $\mathrm{Pdx}$-Cre;CLEG2 mice. A possible explanation for this discrepancy is that signaling activated through secreted Hedgehog ligands elicits both cell-autonomous and paracrine effects, the latter of which are missing in Pdx-Cre;CLEG2 mice. Also, it is possible that ligand-induced changes in mesenchymalepithelial signaling are responsible for increased propensity of the Kras locus to accrue genetic mutations. Qualitative differences in epithelial-specific versus ligand-mediated paracrine pathway activation with regard to Ras activation and PanIN formation would need to be studied in transgenic mice in which temporal and spatial Hedgehog ligand expression can be achieved in adult pancreas.

Our current data suggest that Hedgehog signaling is activated as a consequence of Ras deregulation. Thus, a hierarchical relationship exists between the Ras and Hedgehog pathways in PanIN progression and pancreatic cancer. The observation that the latency period of PanIN and cancer formation is significantly reduced in $P d x$ Cre; CLEG2;Kras ${ }^{G 12 D}$ triple-transgenic mice compared with either Pdx-Cre;CLEG2 or Pdx-Cre;Kras ${ }^{G 12 D}$ animals further indicates that enforced up-regulation of Hedgehog activity promotes tumor progression in concert with aberrant Ras signaling. The exact mechanism by which deregulation of Hedgehog signaling increases tumor progression in $\mathrm{Pdx}-\mathrm{Cre} ; \mathrm{Kras}^{G 12 D}$ animals needs to be investigated. However, the Hedgehog pathway is known to directly activate cell proliferation (Ruiz i Altaba et al. 2002), and these effects might explain the rapid growth of the tumors found in compound mice.

\section{Activation of AKT in Pdx-Cre; CLEG2 tumors}

Results from the current study also shed light on Hedgehog's role in regulating the activity of other signaling pathways in epithelial cells. While activation of the classic Ras/MAPK pathway is not observed in Pdx-Cre; CLEG2 pancreata, the undifferentiated tumors that form in these mice are marked by an activation of the AKT pathway. AKT signaling is a hallmark of many human cancers (for review, see Vivanco and Sawyers 2002), including pancreatic adenocarcinoma (for review, see Hezel et al. 2006), where it promotes tumor progression by triggering responses such as cell proliferation, survival, and motility. Although AKT activation can be mediated through Ras signaling, our results point to regulation via receptor kinase signaling, including the EGFR pathway. Hedgehog signaling has been shown to regulate EGFR expression in Drosophila (for review, see Pasca di Magli- ano and Hebrok 2003). EGFR signaling is prominent in human pancreatic cancer and is also observed in the undifferentiated tumors that form in Pdx-Cre;CLEG2 mice. Thus, our results suggest that epithelial-specific Hedgehog signaling does not promote Ras/MAPK signaling. In contrast, Hedgehog signaling might regulate cell proliferation and survival via activation of EGFR/AKT signaling.

\section{Hedgehog and Notch signaling pathways in pancreatic cancer}

The induction of cell transformation in Pdx-Cre;CLEG2 places the Hedgehog pathway at an early stage of tumor formation. However, previous reports have assigned important roles to other signaling pathways during pancreatic tumor initiation (Pasca di Magliano and Hebrok 2003). The Notch signaling pathway is important during pancreas development, where it promotes self-renewal of pancreatic progenitors while inhibiting progenitor differentiation (Apelqvist et al. 1999; Jensen et al. 2000; Murtaugh et al. 2003; Esni et al. 2004). In the adult pancreas, the Notch pathway is down-regulated, remaining active only in the centroacinar cells and in a small subset of ductal cells (Miyamoto et al. 2003; Stanger et al. 2005). Interestingly, up-regulation of Notch pathway components is observed in PanIN1 lesions and pathway activity remains high in PDA compared with a normal pancreas (Miyamoto et al. 2003). Ectopic expression of TGF- $\alpha$ in pancreatic acinar cells induces activation of the Notch signaling pathway and results in the formation of PanIN lesions, but it is not sufficient to promote progression to pancreatic adenocarcinoma in the absence of Trp $53 \mathrm{mu}-$ tations (Sandgren et al. 1990; Wagner et al. 1998, 2001; Miyamoto et al. 2003). Analysis of the expression of the Notch target gene Hes1 in the Kras ${ }^{G 12 D}$-Trp53 model of pancreatic adenocarcinoma indicated that the Notch pathway is up-regulated in PanIN lesions (Hingorani et al. 2005). Here, we show that up-regulation of Hes1 is observed in tumors of $P d x$-Cre; $C L E G 2$ mice, even in the absence of an activating Kras mutation. These results indicate that up-regulation of the Notch pathway might occur downstream from the Hedgehog pathway during pancreatic adenocarcinoma progression. However, our data do not exclude that Notch signaling might also activate the Hedgehog pathway, as has been observed in other contexts (Androutsellis-Theotokis et al. 2006). Since both the Notch and the Hedgehog signaling pathways have been shown to promote proliferation of progenitor cells, it would be interesting to test whether Notch-mediated tumors also show a poorly differentiated phenotype.

Current knowledge indicates that PDA develops via a defined sequence of signature events, including mutations in tumor suppressors and oncogenes as well as deregulation of embryonic signaling pathways that set the stage for malignant transformation. Our findings support the concept that aberrantly activated Hedgehog signaling cooperates with Kras mutations to drive early stages of pancreatic neoplasia. Defining the exact hierarchical in- 
teractions between these events will be critical for our understanding of the etiology of this disease as well as the potential for therapeutic intervention.

\section{Material and methods}

\section{Mouse strains}

Pdx1-Cre mice (Gu et al. 2002) were intercrossed with CLEG2 mice and with $L S L-K_{r a s}{ }^{G 12 D}$ mice (a gift from David Tuveson, University of Pennsylvania, Philadelphia, PA, referred to as Kras $^{\text {G12D }}$ throughout the text) (Hingorani et al. 2003) to generate either double or triple mutants: Pdx-Cre; CLEG2 and PdxCre;CLEG2;Kras ${ }^{G 12 D}$. All studies were conducted in compliance with University of California IACUC (Institutional Animal Care and Use Committee) guidelines. CLEG2 mice were generated using the ubiquitously expressed $C A G$ promoter (Niwa et al. 1991), followed by a floxed EGFP cDNA and bovine growth hormone polyA sequence, followed by myc-tagged GLI2 $\Delta N$ cDNA (Roessler et al. 2005) and 2xSV40 polyA sequence (A. Ermilov, J. Ferris, and A.A. Dlugosz, in prep.).

\section{Western blotting}

Tissue samples were homogenized in RIPA buffer $(50 \mathrm{mM}$ Tris$\mathrm{HCl}$ at $\mathrm{pH} 7.4,1 \%[\mathrm{v} / \mathrm{v}] \mathrm{NP} 40,0.1 \%[\mathrm{w} / \mathrm{v}]$ SDS, $0.25 \%[\mathrm{w} / \mathrm{v}]$ Na-deoxycholate, $1 \mathrm{mM}$ EDTA, phosphatase [1 mM Na-orthovanadate, $40 \mathrm{mM} \mathrm{NaF}, 10 \mathrm{mM}$ glycerophosphate, $5 \mathrm{mM}$ pyrophosphate], protease inhibitor [Complete-Roche]). Equal amounts of proteins were electrophoresed in 15\% SDS-PAGE gels, transferred to PVDF membrane (Bio-Rad), and processed for immunoblotting with antibodies against ERK1/2, phosphoERK1/2, AKT, phospho-AKT, phospho-PDK1, phospho-s6K (p70), and phospo-EGFR (1:1000 dilution; Cell Signaling Technology) or GAPDH (1:3000 dilution). HRP-conjugated secondary antibody was used at 1:1000 dilution and detected by ECL (Amersham Biosciences).

\section{Histological analysis}

Control pancreas and tumor tissue samples were fixed overnight in zinc-containing neutral-buffered formalin, embedded in paraffin, cut into 5 - $\mu \mathrm{m}$-thick sections, and placed on Superfrost Plus slides (Fisher Scientific). Sections were subjected to hematoxylin and eosin (HE), Periodic Acid Schiff (PAS), Alcian blue, and immunohistochemical staining. For immunohistochemistry, deparaffinized and rehydrated slides were subjected to antigen retrieval via autoclaving in a $10 \mathrm{mM}$ citric acid buffer $(\mathrm{pH}$ 6.0). Upon cooling to room temperature for $30 \mathrm{~min}$ slides were blocked with $0.3 \% \mathrm{H}_{2} \mathrm{O}_{2}$ for 20 min, washed in phosphate-buffered saline (PBS), and then blocked with $1 \%$ BSA in PBS. Slides were incubated with diluted primary antibodies overnight at $4{ }^{\circ} \mathrm{C}$. The following primary antibodies were used: mouse anti$\beta$-catenin (1:200 dilution; Becton and Dickinson), mouse anti- myc (1:100 dilution; Neomarkers), rabbit anti-Hes1 (1:500 dilution; gift from Dr. Tetsuo Sudo, Toray Industries, Inc., Kamakura, Japan), rabbit anti-ki67 (1:200 dilution; Novocastra), mouse anti-E-cadherin (1:200 dilution; Becton and Dickinson), rat anti-CK19 (TROMAIII, 1:1000 dilution; developed by Dr. Rolf Kemler [Max-Planck Institute of Immunobiology, Freiburg, Germany] and obtained from the Hybridoma Bank at the University of Iowa), goat anti-SHH (1:100 dilution; Becton and Dickinson). Biotinylated anti-rabbit (Vector Laboratories), and anti-goat, anti-rat, and anti-mouse (Jackson Immunoresearch) antibodies were used as secondary antibodies at a 1:300 dilution. 3-3'-Diaminobenzidine tetrahydrochloride was used as a chromogen. Bright-field images were acquired using a Zeiss Axio Imager D1 scope.

\section{Quantitative PCR}

Total RNA was prepared from pancreas or tumor tissue samples using RNeasy (Qiagen) according to the manufacturer's protocol. Reverse-transcription reaction was performed using SuperScript III First-Strand Synthesis System (Invitrogen). PCR reactions were performed in a $25-\mu \mathrm{L}$ reaction mixture containing $1 \times$ SYBR Green PCR master mix (Applied Biosystems) and $300 \mathrm{nM}$ of each primer. Amplification was performed by initial polymerase activation for $10 \mathrm{~min}$ at $95^{\circ} \mathrm{C}$, and 40 cycles of $94^{\circ} \mathrm{C}$ for 30 sec followed by elongation for $1 \mathrm{~min}$ at $60^{\circ} \mathrm{C}$. Primer sequences used are listed in Table 1 . To exclude contamination with nonspecific PCR products, melting curve analysis was applied to all final PCR products after the cycling protocol. RNA samples without reverse-transcription were also subjected to PCR reaction to exclude contamination of genomic DNA. Expression of Hedgehog pathway genes was compared with the expression level of $\beta$-glucuronidase (GUS) as previously described (Sekine et al. 2006).

\section{Acknowledgments}

We are indebted to Dr. Doug Melton for providing the Pdx-Cre mouse strain and Drs. David Tuveson and Sunil Hingorani for sharing the Kras ${ }^{G 12 D}$ mice; Dr. Jun-ichi Miyazaki for providing the CAG promoter; Drs. Erich Roessler and Maximilian Muenke for providing myc-tagged GLI2 $\Delta N$ cDNA; and Dr. Tetsuo Sudo for sharing the anti-Hesl antibody. The anti-CK19 antibody (TROMAIII) developed by Dr. Rolf Kemler (MaxPlanck Institute of Immunobiology, Freiburg, Germany) was obtained from the Developmental Studies Hybridoma Bank developed under the auspices of the NICHD and maintained by The University of Iowa, Department of Biological Sciences, Iowa City, IA. We would also like to thank John P. Morris IV and Drs. Brian Lewis and Roman Nawroth for critical reading of the manuscript. The work in M.H.'s laboratory was supported by grants from the NIH (DK60533-01A1, CA112537-01, and P30 CA82103); image acquisition was supported by the University of California at San Francisco Diabetes and Endocrinology Re-

Table 1. Quantitative PCR primers

\begin{tabular}{|c|c|c|}
\hline & Forward primer & Reverse primer \\
\hline Shh & CAAAGCTCACATCСACTGTTCTG & GAAACAGCCGCCGGATTT \\
\hline Ihh & CACGTGCATTGCTCTGTCAA & AGGAAAGCAGCCACCTGTCTT \\
\hline Ptc1 & TTGTGGAAGCCACAGAAAACC & TGTCTGGAGTCCGGATGGA \\
\hline Gli1 & TGGACTCTCTTGACCTGGACAAC & GGCCCTGGGCCTCATC \\
\hline GUS & ACGGGATTGTGGTCATCGA & TCGTTGCCAAAACTCTGAGGTA \\
\hline
\end{tabular}


search Center Microscopy Core (P30 DK63720). Work in A.A.D.'s laboratory was supported by NIH grants CA87837 and AR45973. M.P. was supported by a fellowship grant from the National Pancreas Foundation.

\section{References}

Aguirre, A.J., Bardeesy, N., Sinha, M., Lopez, L., Tuveson, D.A., Horner, J., Redston, M.S., and DePinho, R.A. 2003. Activated Kras and Ink4a/Arf deficiency cooperate to produce metastatic pancreatic ductal adenocarcinoma. Genes \& Dev. 17: 3112-3126.

Almoguera, C., Shibata, D., Forrester, K., Martin, J., Arnheim, N., and Perucho, M. 1988. Most human carcinomas of the exocrine pancreas contain mutant c-K-ras genes. Cell 53: $549-554$.

Androutsellis-Theotokis, A., Leker, R.R., Soldner, F., Hoeppner, D.J., Ravin, R., Poser, S.W., Rueger, M.A., Bae, S.K., Kittappa, R., and McKay, R.D. 2006. Notch signalling regulates stem cell numbers in vitro and in vivo. Nature 442: 823-826.

Apelqvist, A., Ahlgren, U., and Edlund, H. 1997. Sonic hedgehog directs specialised mesoderm differentiation in the intestine and pancreas. Curr. Biol. 7: 801-804.

Apelqvist, A., Li, H., Sommer, L., Beatus, P., Anderson, D.J., Honjo, T., Hrabe de Angelis, M., Lendahl, U., and Edlund, H. 1999. Notch signalling controls pancreatic cell differentiation. Nature 400: 877-881.

Berman, D.M., Karhadkar, S.S., Maitra, A., Montes de Oca, R., Gerstenblith, M.R., Briggs, K., Parker, A.R., Shimada, Y., Eshleman, J.R., Watkins, D.N., et al. 2003. Widespread requirement for Hedgehog ligand stimulation in growth of digestive tract tumours. Nature 425: 846-851.

Boschman, C.R., Stryker, S., Reddy, J.K., and Rao, M.S. 1994 Expression of p53 protein in precursor lesions and adenocarcinoma of human pancreas. Am. J. Pathol. 145: 1291-1295.

Deramaudt, T.B., Takaoka, M., Upadhyay, R., Bowser, M.J., Porter, J., Lee, A., Rhoades, B., Johnstone, C.N., Weissleder, R., Hingorani, S.R., et al. 2006. N-cadherin and keratinocyte growth factor receptor mediate the functional interplay between $\mathrm{Ki}-R A S^{G 12 V}$ and $p 53^{V 143 A}$ in promoting pancreatic cell migration, invasion, and tissue architecture disruption. Mol. Cell. Biol. 26: 4185-4200.

Esni, F., Ghosh, B., Biankin, A.V., Lin, J.W., Albert, M.A., Yu, X., MacDonald, R.J., Civin, C.I., Real, F.X., Pack, M.A., et al. 2004. Notch inhibits Ptf1 function and acinar cell differentiation in developing mouse and zebrafish pancreas. Development 131: 4213-4224.

Gu, G., Dubauskaite, J., and Melton, D.A. 2002. Direct evidence for the pancreatic lineage: NGN3+ cells are islet progenitors and are distinct from duct progenitors. Development 129: 2447-2457.

Hahn, S.A., Schutte, M., Hoque, A.T.M.S., Moskaluk, C.A., da Costa, L.T., Rozenblum, E., Weinstein, C.L., Fischer, A., Yeo, C.J., Hruban, R.H., et al. 1996. DPC4, a candidate tumor suppressor gene at human chromosome 18q21.1. Science 271: 350-353.

Hezel, A.F., Kimmelman, A.C., Stanger, B.Z., Bardeesy, N., and Depinho, R.A. 2006. Genetics and biology of pancreatic ductal adenocarcinoma. Genes \& Dev. 20: 1218-1249.

Hingorani, S.R., Petricoin, E.F., Maitra, A., Rajapakse, V., King, C., Jacobetz, M.A., Ross, S., Conrads, T.P., Veenstra, T.D., Hitt, B.A., et al. 2003. Preinvasive and invasive ductal pancreatic cancer and its early detection in the mouse. Cancer Cell 4: 437-450.

Hingorani, S.R., Wang, L., Multani, A.S., Combs, C., Deram- audt, T.B., Hruban, R.H., Rustgi, A.K., Chang, S., and Tuveson, D.A. 2005. Trp53 ${ }^{R 172 H}$ and $\operatorname{Kras}^{G 12 D}$ cooperate to promote chromosomal instability and widely metastatic pancreatic ductal adenocarcinoma in mice. Cancer Cell 7: 469-483.

Hoorens, A., Prenzel, K., Lemoine, N.R., and Kloppel, G. 1998. Undifferentiated carcinoma of the pancreas: Analysis of intermediate filament profile and Ki-ras mutations provides evidence of a ductal origin. J. Pathol. 185: 53-60.

Hruban, R.H., Adsay, N.V., Albores-Saavedra, J., Compton, C., Garrett, E.S., Goodman, S.N., Kern, S.E., Klimstra, D.S., Kloppel, G., Longnecker, D.S., et al. 2001. Pancreatic intraepithelial neoplasia: A new nomenclature and classification system for pancreatic duct lesions. Am. J. Surg. Pathol. 25: 579-586.

Hruban, R.H., Adsay, N.V., Albores-Saavedra, J., Anver, M.R., Biankin, A.V., Boivin, G.P., Furth, E.E., Furukawa, T., Klein, A., Klimstra, D.S., et al. 2006. Pathology of genetically engineered mouse models of pancreatic exocrine cancer: Consensus report and recommendations. Cancer Res. 66: 95106.

Jemal, A., Siegel, R., Ward, E., Murray, T., Xu, J., Smigal, C., and Thun, M.J. 2006. Cancer statistics, 2006. CA Cancer J. Clin. 56: $106-130$

Jensen, J., Pedersen, E.E., Galante, P., Hald, J., Heller, R.S., Ishibashi, M., Kageyama, R., Guillemot, F., Serup, P., and Madsen, O.D. 2000. Control of endodermal endocrine development by Hes-1. Nat. Genet. 24: 36-44.

Kawahira, H., Scheel, D.W., Smith, S.B., German, M.S., and Hebrok, M. 2005. Hedgehog signaling regulates expansion of pancreatic epithelial cells. Dev. Biol. 280: 111-121.

Klimstra, D.S. and Longnecker, D.S. 1994. K-ras mutations in pancreatic ductal proliferative lesions. Am. J. Pathol. 145: $1547-1550$.

Lammert, E., Brown, J., and Melton, D.A. 2000. Notch gene expression during pancreatic organogenesis. Mech. Dev. 94: 199-203.

Maitra, A., Adsay, N.V., Argani, P., Iacobuzio-Donahue, C., De Marzo, A., Cameron, J.L., Yeo, C.J., and Hruban, R.H. 2003. Multicomponent analysis of the pancreatic adenocarcinoma progression model using a pancreatic intraepithelial neoplasia tissue microarray. Mod. Pathol. 16: 902-912.

Miyamoto, Y., Maitra, A., Ghosh, B., Zechner, U., Argani, P., Iacobuzio-Donahue, C.A., Sriuranpong, V., Iso, T., Meszoely, I.M., Wolfe, M.S., et al. 2003. Notch mediates TGF $\alpha$-induced changes in epithelial differentiation during pancreatic tumorigenesis. Cancer Cell 3: 565-576.

Moskaluk, C.A., Hruban, R.H., and Kern, S.E. 1997. p16 and $\mathrm{K}$-ras gene mutations in the intraductal precursors of human pancreatic adenocarcinoma. Cancer Res. 57: 2140-2143.

Murtaugh, L.C., Stanger, B.Z., Kwan, K.M., and Melton, D.A. 2003. Notch signaling controls multiple steps of pancreatic differentiation. Proc. Natl. Acad. Sci. 100: 14920-14925.

Niwa, H., Yamamura, K., and Miyazaki, J. 1991. Efficient selection for high-expression transfectants with a novel eukaryotic vector. Gene 108: 193-200.

Pasca di Magliano, M. and Hebrok, M. 2003. Hedgehog signaling in cancer formation and maintenance. Nat. Rev. Cancer 3: 903-911.

Roessler, E., Ermilov, A.N., Grange, D.K., Wang, A., Grachtchouk, M., Dlugosz, A.A., and Muenke, M. 2005. A previously unidentified amino terminal domain regulates transcriptional activity of wild-type and disease-associated human GLI2. Hum. Mol. Genet. 14: 2181-2188.

Rozenblum, E., Schutte, M., Goggins, M., Hahn, S.A., Panzer, S., Zahurak, M., Goodman, S.N., Sohn, T.A., Hruban, R.H., 
Yeo, C.J., et al. 1997. Tumor-suppressive pathways in pancreatic carcinoma. Cancer Res. 57: 1731-1734.

Ruiz i Altaba, A., Sanchez, P., and Dahmane, N. 2002. Gli and Hedgehog in cancer: Tumours, embryos and stem cells. Nat. Rev. Cancer 2: 361-372.

Sandgren, E.P., Luetteke, N.C., Palmiter, R.D., Brinster, R.L., and Lee, D.C. 1990. Overexpression of TGF $\alpha$ in transgenic mice: Induction of epithelial hyperplasia, pancreatic metaplasia, and carcinoma of the breast. Cell 61: 1121-1135.

Sekine, S., Lan, B.Y., Bedolli, M., Feng, S., and Hebrok, M. 2006. Liver-specific loss of $\beta$-catenin blocks glutamine synthesis pathway activity and cytochrome p450 expression in mice. Hepatology 43: 817-825.

Stanger, B.Z., Stiles, B., Lauwers, G.Y., Bardeesy, N., Mendoza, M., Wang, Y., Greenwood, A., Cheng, K.H., McLaughlin, M., Brown, D., et al. 2005. Pten constrains centroacinar cell expansion and malignant transformation in the pancreas. Cancer Cell 8: 185-195.

Thayer, S.P., di Magliano, M.P., Heiser, P.W., Nielsen, C.M., Roberts, D.J., Lauwers, G.Y., Qi, Y.P., Gysin, S., Fernandezdel Castillo, C., Yajnik, V., et al. 2003. Hedgehog is an early and late mediator of pancreatic cancer tumorigenesis. $\mathrm{Na}$ ture 425: 851-856.

Vivanco, I. and Sawyers, C.L. 2002. The phosphatidylinositol 3-Kinase AKT pathway in human cancer. Nat. Rev. Cancer 2: 489-501.

Wagner, M., Luhrs, H., Kloppel, G., Adler, G., and Schmid, R.M. 1998. Malignant transformation of duct-like cells originating from acini in transforming growth factor transgenic mice. Gastroenterology 115: 1254-1262.

Wagner, M., Greten, F.R., Weber, C.K., Koschnick, S., Mattfeldt, T., Deppert, W., Kern, H., Adler, G., and Schmid, R.M. 2001. A murine tumor progression model for pancreatic cancer recapitulating the genetic alterations of the human disease. Genes \& Dev. 15: 286-293.

Wilentz, R.E., Iacobuzio-Donahue, C.A., Argani, P., McCarthy, D.M., Parsons, J.L., Yeo, C.J., Kern, S.E., and Hruban, R.H. 2000. Loss of expression of Dpc4 in pancreatic intraepithelial neoplasia: Evidence that DPC4 inactivation occurs late in neoplastic progression. Cancer Res. 60: 2002-2006. 


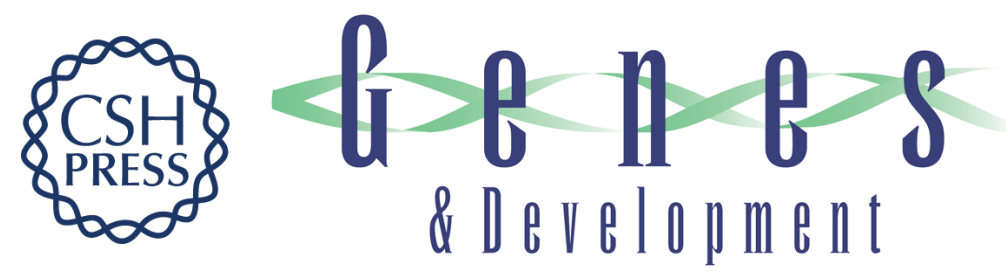

\section{Hedgehog/Ras interactions regulate early stages of pancreatic cancer}

Marina Pasca di Magliano, Shigeki Sekine, Alexandre Ermilov, et al.

Genes Dev. 2006, 20:

Access the most recent version at doi:10.1101/gad.1470806

Supplemental

Material

References

License

Email Alerting Service
http://genesdev.cshlp.org/content/suppl/2006/10/26/20.22.3161.DC1

This article cites 39 articles, 11 of which can be accessed free at: http://genesdev.cshlp.org/content/20/22/3161.full.html\#ref-list-1

Receive free email alerts when new articles cite this article - sign up in the box at the top right corner of the article or click here.

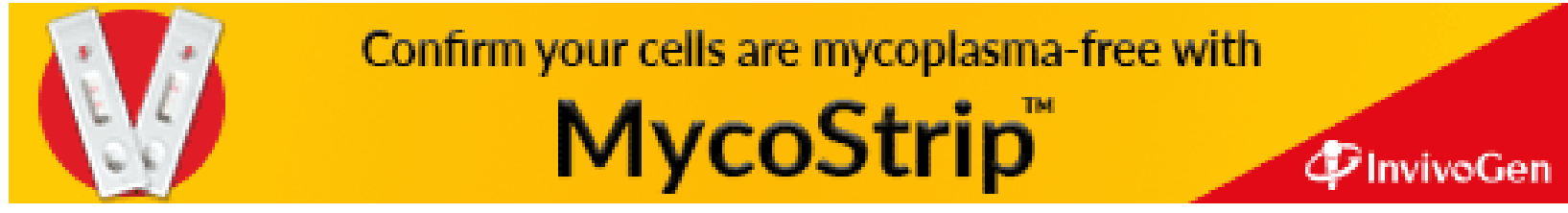

